\title{
MEJORAMIENTO DEL DESEMPEÑO TÉRMICO DE SISTEMAS DE CONSTRUCCIÓN NO CONUENCIONAL EN EL NEA: EUALUACIÓN Y PROPUESTA DE ATENUACIÓN DE PUENTES TÉRMICOS
}

\author{
Manuel VENHAUS HELD ${ }^{1}$; Herminia M. ALÍAS ${ }^{2}$; Guillermo JACOBO ${ }^{3}$ \\ manu_ven@hotmail.com \\ 1. Becario de Investigación de Pregrado, Secretaría General de Ciencia y Técnica (SGCyT), UNNE. \\ 2. Directora beca pregrado SGCYT, UNNE, profesora adjunta cátedra Estructuras II (FAU-UNNE). \\ 3. Director de proyecto SGCyT, UNNE, profesor titular cátedra Estructuras II (FAU-UNNE).
}

\section{PALABRAS CLAUE}

Transmitancia térmica; construcción industrializada; software.

\section{RESUMEN}

El buen comportamiento higrotérmico de las envolventes perimetrales de edificios construidos en el Nordeste Argentino (NEA) resulta de primordial importancia, dado el clima la región registra. No obstante, este se ve desmejorado debido a la gran cantidad de heterogeneidades (puentes térmicos) que la naturaleza estructural de los sistemas de construcción no convencional genera; esto posibilita un mayor flujo de calor a través de las secciones transversales. Teniendo en cuenta que únicamente el $20 \%$ de este tipo de soluciones constructivas resulta apto ante este punto crítico intrínseco, el presente trabajo propone su evaluación y atenuación mediante pautas de diseño.

\section{KEYWORDS}

Thermal transmittance; industrial building; software

\section{ABSTRACT}

The good hygrothermal behavior of the perimetric envelopes of buildings constructed in the Argentine Northeast (NEA) is of paramount importance, given the climate the region registers. However, this is undermined by the large number of heterogeneities (thermal bridges) that the structural nature of non-conventional construction systems generates; This enables a greater flow of heat through the cross sections. Taking into account that only $20 \%$ of this type of constructive solutions is suitable for this intrinsic critical point, the present work proposes its evaluation and attenuation through design guidelines. 


\section{OBJETIUOS}

El presente trabajo tiene como principal objetivo analizar, evaluar, diagnosticar y proponer criterios para mejorar térmicamente las envolventes exteriores de edificios materializados mediante la tecnología y los materiales de la construcción no convencional, en los principales centros urbanos del NEA. Para ello parte de la especial consideración del impacto que tienen los puentes térmicos de los cerramientos exteriores, verdaderos puntos críticos que se extienden por toda la envolvente, de modo de lograr que los espacios interiores que ellas albergan reúnan condiciones higrotérmicas más cercanas a las del confort y, por lo tanto, demanden un uso menos intensivo de dispositivos electromecánicos de climatización artificial, con la consecuente disminución del consumo de energía eléctrica.

\section{INTRODUCCIÓN}

Una primera aproximación define los sistemas constructivos no tradicionales o no convencionales como aquellosque porelempleo de materiales o técnicas novedosas no son tan conocidos y su uso no se encuentra muy difundido en una determinada región. Por otro lado, se los podría definir como aquellos que emplean materiales prefabricados elaborados en serie con maquinaria automatizada y que, porello, requieren equipos y mano de obra especializada o profesional para su manipulación. La diferenciación entre "convencional" y "no convencional", en definitiva, depende de la consideración de la localización y el momento histórico en que se desarrolla un determinado sistema constructivo. De esta manera, puede una misma técnica ser tradicional en una región y no tradicional en otra, o transformarse en tradicional con el paso del tiempo.

A los fines de este trabajo y en concordancia con el consenso generalizado sobre la igualdad entre estos términos, se considera a la construcción "no convencional" como aquella "producción de edificios reemplazando la mano de obra artesanal, con máquinas utilizadas por obreros especializados en su manejo, o con máquinas automáticas"1. La bibliografía ubica a los primeros antecedentes de construcción no convencional en el siglo XVI, y a partir del siglo XIX las diferentes técnicas se desarrollaron de manera acelerada en Europa y Estados Unidos, con el propósito de optimizar la eficiencia de los procesos productivos de la industria de la construcción. No obstante, en la región del NEA, su implementación aún no es muy frecuente, aunque incrementó notablemente en los últimos años y se observa un notable aumento.

Los sistemas constructivos no convencionales de mayor difusión y uso para la materialización de la envolvente de edificios en esta región se caracterizan casi con exclusividad por la construcción en seco, a través de algunas de las formas de los sistemas de entramado y emplacado. En general, la estructura portante de los edificios así materializados constituye importantes puentes térmicos. Los puentes térmicos pueden ser definidos como "heterogeneidades de un elemento constructivo que forman parte de la envolvente (pared, piso, techo, etc.) de un edificio que ocasionan

1. Estudio-Diagnóstico sobre las posibilidades del desarrollo de una edificación residencial industrializada dirigida a satisfacer las necesidades de vivienda pública y muy especialmente en alquiler en la Comunidad Autónoma del País Vasco.

2. Norma IRAM 11.549. Aislamiento térmico de edificios. Vocabulario. Tercera Edición. Instituto Argentino de Racionalización de Materiales. Buenos Aires, Argentina. 2002

mayor flujo de calor a través de esta"2. Estas discontinuidades en una superficie de cerramiento presentan menor resistencia térmica que el resto de dicho cerramiento y por elloocasionan comportamientos estructurales, físicos, mecánicos e higrotérmicos diferenciales, que frecuentemente causan patologías constructivas.

Si consideramos que los edificios son responsables de más del $40 \%$ de la energía consumida en nuestro país, que de esa energía la mayor parte es insumida en climatización, es decir, calefacción y refrigeración de los ambientes interiores, y que el $50 \%$ de esta energía es ganada o perdida a través de los cerramientos opacos del edificio, sumado a que el clima de nuestra zona bioambiental (zona "I", subzona "b", según norma IRAM 11603) es muy cálido, con altas temperaturas en verano e inviernos moderados, con altas humedades relativas, que dan lugar a sensaciones psicofísicas de disconfort en los usuarios de los edificios, resultaría muy importante y necesario aumentar la eficiencia energética de los edificios a través del mejoramiento del desempeño higrotérmico de sus envolventes.

\section{DESARROLLO Y RESULTTADOS}

En una primera instancia se relevaron y estudiaron los principales tipos y subtipos de construcciones no convencionales y los materiales empleados para su construcción. De estos tipos se determinaron y describieron aquellosque de manera más usual son empleados en el NEA.

\section{Tipos y subtipos de construcción no convencional}

Como principal exponente de los sistemas livianos se reconoció el sistema liviano con entramado de madera. Este subtipo, tam- 
bién llamado balloon framing, está formado por la combinación de elementos portantes ligeros de madera, comúnmente denominados soleras y montantes, distanciados entre 0,40 y $0,60 \mathrm{~m}$ para formar bastidores portantes. Asimismo, se reconoció el uso extendido del sistema liviano con entramado metálico. Este deriva del primero y emplea para la constitución de los entramados perfiles metálicos galvanizados conformados en frío con forma de "C" o "U". En ambos casos los bastidores que sirven de estructura se completan con variados elementos de cerramiento y revestimiento, empleados como acabado y protección. Dentro de este tipo de construcción no convencional se reconoció también la aplicación del sistema liviano de paneles prefabricados. Son paneles de tipo sándwich con estructura de madera quecomponen lostabiquesinteriores, exteriores y cielorrasos. La estructura interna de los paneles se materializa con bastidores de madera de pino, y su terminación interior es de placas de roca de yeso. La terminación exterior varía de acuerdo con la marca comercial y se materializa por lo general con placas cementicias de alto impacto, pudiendo incluso conformarse con mamposterías, que dan una imagen más tradicional. Por el lado de los sistemas pesados, se detectó el uso del sistema de grandes paneles, con paneles transversales de carga de hormigón armado espaciados a 3,00 m y paneles rigidizantes en la parte central de los edificios (MAC DonneL, 1999). Las terminaciones interiores se materializan con placas de roca de yeso sobre estructuras metálicas.

Dentrodelgrupodesistemasindustrializados in situ, se analizó como exponente principal al sistema de paneles aligerados con núcleo de poliestireno expandido y malla electro-soldada espacial. Este tipo de sistema industrializado abierto utiliza paneles de poliestireno expandido y mallas de acero prefabricados en forma modular, que luego reciben la aplicación a presión de un revoque estructural de hormigón en obra, que provee estructura, protección y terminación. Los cinco sistemas constructivos reconocidos como habituales en el NEA seconstituyenen multicapasque incorporan en su interior la aislación hidráulica, térmica y acústica. Pero, debido a su naturaleza constructiva, la repetición de piezas estructurales para la conformación de los bastidores y estructuras portantes interrumpe el normal desarrollo de las aislaciones térmicas en las multicapas y conforma las heterogeneidades denominadas puentes térmicos. Contemplando los diferentes materiales, escuadrías y formas que adoptan estos en los sistemas constructivos antes descriptos, puede decirse que representan alrededor del $10 \%$ del desarrollo normal de las envolventes.

La imagen 1 ilustra un esquema de conformación de la estructura del sistema liviano de entramado metálico. no obstante, similares esquemas pueden aplicarse a otro tipo de sistemas y materiales antes descriptos. Se desea mostrar la importante presencia de elementos estructurales intermedios que presenta la construcción no convencional y en especial en seco.

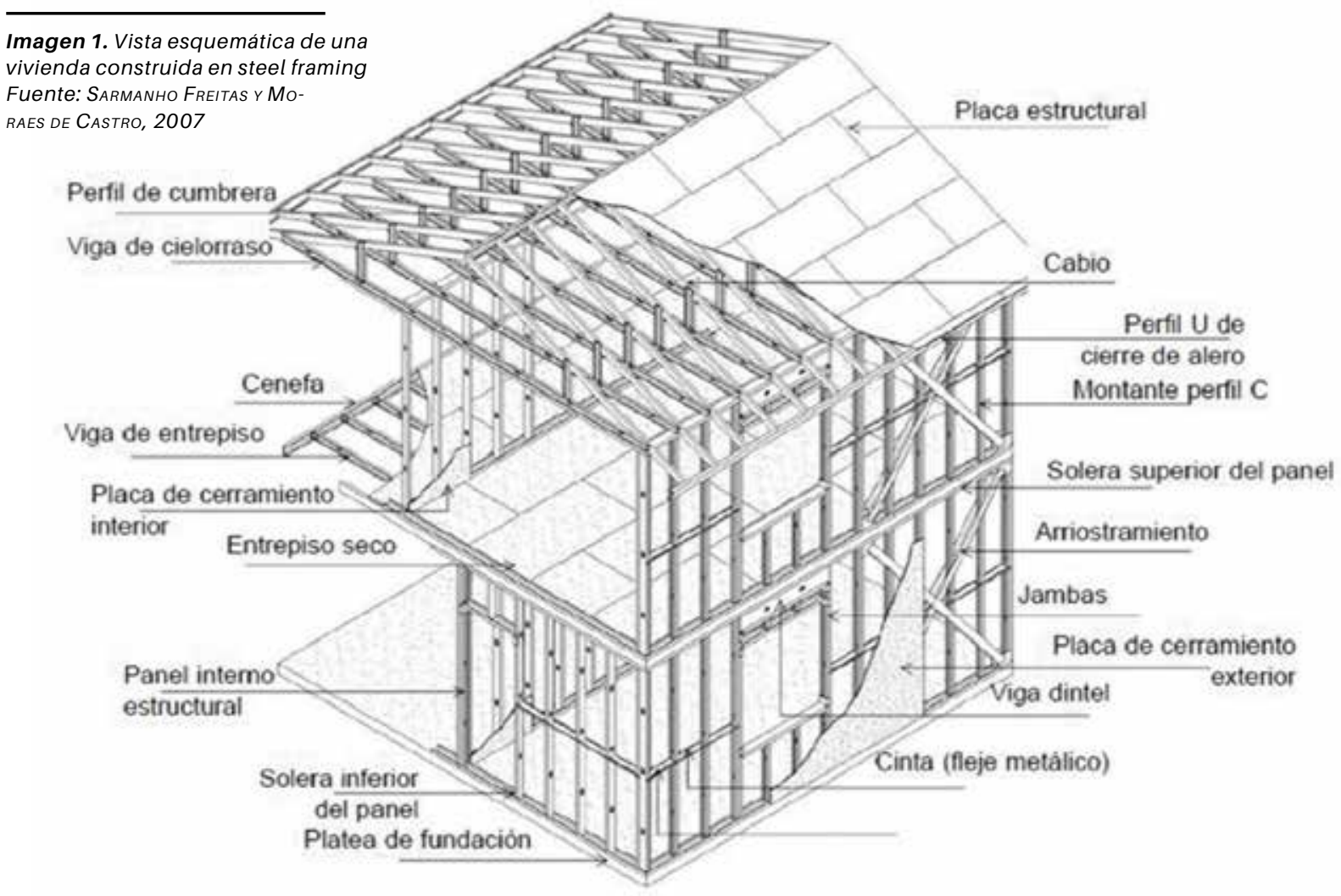




\section{Normas técnicas vigentes}

Para analizar la influencia de estos en el comportamiento general de los sistemas constructivos antes descriptos, se los evaluó de acuerdo con tres criterios. Los principales criterios establecidos por los reglamentos generales de construcción y códigos de edificación de los centros urbanos más importantes de la región fueron estudiados a fin de efectuar una evaluación del cumplimiento de las condiciones técnico-constructivas que ellos establecen para la construcción no convencional. Al respecto, puede mencionarse que se avocan en profundidad a la reglamentación del diseño de ambientes (dimensiones, iluminación, ventilación), la imagen de las obras y la conformación urbana que surge del conjunto de ellas. En cuanto a sistemas o materiales constructivos no convencionales, sin embargo, presentan una definición, clasificación y prescripción escueta y poco detallada. El Reglamento General de Construcciones de la Municipalidad de Resistencia y el Código de Edificación de la Ciudad de Corrientes contemplan el uso de variados sistemas de construcción, siempre que cuenten con Certificado de Aptitud Técnica (CAT). Este es un documento de aprobación otorgado por la Subsecretaría de Vivienda y Desarrollo Ambiental de la Nación a todo material, elemento o sistema constructivo que considere "no tradicional". Para su obtención se demanda un informe detallado de materias primas y procesos de fabricación empleados, así como la aplicación final de los materiales, componentes o sistemas. Asimismo, se exige la presentación de ensayos de conductividad térmica y resistencia al paso de vapor de agua.
Por su parte, el Código de Edificación de la Municipalidad de Posadas detalla un mecanismo propio de certificación de aptitud técnica de materiales, equipos y sistemas constructivos no convencionales. Usualmente, las certificaciones antes mencionadas deben ser renovadas cada tres años, lo cual garantiza la revisión y optimización constante de los sistemas constructivos. Finalmente, el Reglamento General de Construcciones de la Ciudad de Formosa, si bien contempla la construcción de estructuras de hormigón armado, metal o madera con las pertinentes protecciones, es el que menor mención hace a la construcción no tradicional.

\section{Normas de habitabilidad vigentes}

Los procedimientos establecidos por la Normativa de Habitabilidad del Instituto Argentino de Normalización y Certificación (IRAM), las Normas IRAM de la serie 11.600, fueron empleados para evaluar los diferentes sistemas constructivos en estudio. En primer lugar, se determinó el coeficiente de transmitancia térmica (K) para cada sistema de acuerdo con los métodos de cálculo del aislamiento térmico de edificios propuestos por la Norma IRAM 11.601. El método procede de manera individualizada en el cálculo de la transmitancia térmica lineal de la porción opaca del muro "Kmo" y de la porción de las heterogeneidades "Kpt" (puentes térmicos). Estos valores pueden compararse con las transmitancias térmicas máximas admisibles establecidas por la Norma IRAM 11.605 para cada una de las zonas bioambientales en que divide el país. A partir de los valores obtenidos de estas verificaciones se pudo determinar que el $80 \%$ de los sistemas constructivos analizados alcanza para verano el nivel $A$, es decir, el recomendado para laszonas bioambientales Iy II, en que se inscribe el NEA. Para la situación de invierno, el $40 \%$ cumple con el nivel A y el 60 $\%$ restante, con el nivel B (medio).

Una vez establecidas las transmitancias térmicas lineales de ambas porciones del cerramiento, se analiza la relación entre sus valores (Kpt/ Kmo) para determinar la aptitud del cerramiento respecto de los puentes térmicos. Esta se logra si la transmitancia térmica de un puente térmico no supera en más del 50 \% el valor de transmitancia térmica del muro opaco, es decir, $K p t / K m o \leq 1,5$. En casos especiales, en que la distancia entre los puentes térmicos lineales es menor a 1,70 m - caso general de los bastidores de los sistemas constructivos no convencionales-, la tolerancia se reduce al $35 \%$ (Kpt/ $K m o \leq 1,35)$. En este aspecto, el 80 $\%$ de los sistemas constructivos resultó insatisfactorio. Se puede reconocer la tendencia de disminución de la aptitud de los sistemas constructivos no convencionales respecto de los puentes térmicos cuanto menor es la transmitancia térmica de sus muros opacos. Esto sucede debido a que los materiales aislantes térmicos reducen en gran medida la transmitancia térmica, mientras para los puentes térmicos se emplean materiales con altos coeficientes de conductividad térmica.

Asimismo, se consideraron los riesgos de condensación superficial e Intersticial en la estación de invierno de las distintas soluciones tecnológicas de acuerdo con la 
metodología propuesta por la Norma IRAM 11.625. Para estos cálculos se adoptó la temperatura exterior de diseño de la ciudad de Resistencia $\left(-1,8^{\circ} \mathrm{C}\right)$, la temperatura ideal de diseño interior determinada por la norma en $18^{\circ} \mathrm{C}$ y una humedad relativa interior de $75 \%$. Además, se consideraron los coeficientes de transmitancia térmica $(\mathrm{K})$ obtenidos anteriormente y la resistencia al paso de vapor de agua de cada una de las capas constitutivas. Ninguno de los sistemas constructivos evaluados presenta riesgo de producir condensaciones superficiales en condiciones de invierno, debido a los buenos valores de resistencia térmica que se pudieron obtener. No obstante, cada uno de ellos registró riesgo de condensación intersticial, por lo general a partir del material aislante hacia las capas constitutivas exteriores del cerramiento. Esto puede explicarse a partir de la drástica reducción de temperatura de bulbo seco que provocan los diferentes materiales aislantes térmicos y el escaso freno al vapor de agua que ejercen. La consecuente aparición de agua en las capas intersticiales de los cerramientos edilicios altera sus propiedades físicas y químicas y pone en riesgo su durabilidad, comportamiento mecánico e higrotérmico.

\section{Simulaciones con THERM 6.3}

Los diferentes cerramientos tipológicos de los sistemas constructivos estudiados fueron finalmente analizados a partir de simulaciones desarrolladas por el software THERM, en su versión 6.3. Este es un programa de cálculo de calor en régimen estacionario que resuelve numéricamente la ecuación de transmisión de energía en dos dimensiones a través de la sección transversal de elementos de construcción, como ventanas, paredes, cimientos, techos y puertas $^{3}$. El software, desarrollado en Estados Unidos, se encuentra disponible para su descarga gratuita, únicamente en inglés. Utiliza para el desarrollo de los cálculos el método de elementos finitos, un procedimiento numérico empleado en la resolución de problemas que involucran alto grado de complejidad, que incluye geometrías complejas, cargas no distribuidas y la determinación de propiedades materiales. El método propone la resolución de los problemas planteando una serie de ecuaciones algebraicas simultáneas y arroja valores aproximados de incógnitas en un número finito de localizaciones dentro del cuerpo. Los resultados obtenibles de las simulaciones incluyen valores numéricos de transmitancia térmica (U-factors) einformacióngráficacomo vectores de flujo (Flux Vectors) o caminos preponderantes seguidos por elcalor, isotermas (Isotherms) o líneas de igual temperatura y la transmisión del flujo de calor mediante escala de colores (Color Infrared).

Para iniciar con las simulaciones se dibujaron las geometrías de la sección transversal de los cerramientos tipológicos en THERM con la incorporación de plantillas.DFX de base elaboradas con el software de diseño AutoCAD ${ }^{T M}$. A cada geometría luego se le asignó el material correspondiente, extraído de una librería de materiales creada con los mismos valores de conductividad térmica empleados en los cálculos de acuerdo con IRAM. Las simulaciones se realizaron considerando una situación de verano, que afecta en mayor medida al NEA, con un flujo de calor desde el exterior hacia el interior, en sentido horizontal por haber estudiado en detalle los cerramientos verticales. De acuerdo con estas premisas, se determinaron las condiciones de contorno o Boundary Conditions del modelo simulado aplicando para el exterior una temperatura máxima de diseño establecida por la Norma IRAM 11.603 en $39,8^{\circ} \mathrm{C}$ para resistencia y una conductancia de la capa superficial de aire de 25,00 $\mathrm{W} / \mathrm{m}^{2} \mathrm{~K}$, correspondiente a los 0,04 $\mathrm{m}^{2} \mathrm{~K} / \mathrm{W}$ de resistencia superficial exterior (Rse). Como condiciones interiores se adoptó una temperatura ideal de diseño interior de $25^{\circ}$ C para verano y $7,69 \mathrm{~W} / \mathrm{m}^{2} \mathrm{~K}$ como conductancia de la capa superficial de aire, equivalente a los $0,13 \mathrm{~m}^{2} \mathrm{~K} / \mathrm{W}$ de resistencia superficial interior ( $R s i)$ establecida por IRAM. Determinados todos estos parámetros, se procedió a la simulación de los cinco sistemas constructivos no convencionales seleccionados.

Es importante destacar que el software THERM calcula la transmitancia térmica total del modelo dibujado. Esto significa que, si en un mismo modelo se simula el comportamiento de la sección transversal de un determinado sistema constructivo incorporando tanto el muro opaco como el puente térmico, se obtienen gráficos que muestran de forma clara la influencia de las heterogeneidades en la transmisión de calor a través 
de los cerramientos. De esta manera, es asimismo posible obtener un coeficiente ponderado de transmitancia térmica, que representaría la "transmitancia promedio" del cerramiento, teniendo en cuenta la importante presencia de puentes térmicos en la construcción no convencional. Este procedimiento, no obstante, impide establecer las relaciones entre muro opaco y puente térmico (Kpt/Kmo), de acuerdo con la normativa de habitabilidad vigente descripta anteriormente, y determinar a partir de ella la aptitud del cerramiento respecto de los puentes térmicos. Tales consideraciones demandan la simulación de ambas porciones en modelos separados, lo cual, desde el punto de vista gráfico, resulta poco interesante, ya que la riqueza ilustrativa se pierde por la transmisión homogénea de calor que se evalúa. Debido a estas cuestiones, se simularon con THERM 6.3 para cada sistema constructivo un modelo de muro opaco aislado, otro únicamente con la porción de puente térmico y un último que incorpora ambos.

Si de los valores numéricos de transmitancia térmica se trata, si se comparan aquellos obtenidos mediante THERM con los calculadoscon IRAM, se registra una gran similitud en los resultados, con una discrepancia promedio de $3,4 \%$ que se acrecienta conforme se complejiza la figura de la sección transversal. Esto implica que la categorización (nivel A - nivel $B$ - nivel C) de acuerdo con las zonas bioambientales y la aptitud respecto de puentes térmicos $(\mathrm{Kpt} / \mathrm{Kmo} \leq$ $1,35)$ se mantienen. Si, en cambio, se considera la transmitancia térmica ponderada, los rendimientos de los sistemas constructivos disminuyen considerablemente. Comparandolos valores con los que IRAM considera admisibles, el $40 \%$ de los sistemas constructivos alcanzaría el nivel A para verano y únicamente el $20 \%$ el mismonivel para invierno, ubicándose en el nivel B el $40 \%$ para verano y el $60 \%$ para invierno. El sistema de núcleo de poliestireno expandido con malla electro-soldada incluso se encontraría por encima de los niveles mínimos establecidos por norma con esta nueva consideración. Esto denota los errores en que se incurre al considerar únicamente los valores de transmitancia térmica del muro opaco para la categorización, olvidando la importante influencia de los puentes térmicos en el comportamiento general de los entramados estructurales.

Si se analizan en detalle los resultados gráficos obtenidos con THERM, puede advertirse en primer lugar que la mayor reducción de temperatura se produce en la porción correspondiente al aislante térmico, tal como se espera dada su función en la multicapa. Pero, que al verse este interrumpido por un elemento estructural (puente térmico), las líneas isotermas tienden a bordear la heterogeneidad se acercan a los límites del cerramiento. Por otro lado, se reconoce la concentración de vectores de flujoy, por lo tanto, de la transmisión del calor en los puentes térmicos, lo que demuestra asimismo el poder de atracción del flujo que tienen sobre sectores de muro opaco. Si bien los riesgos de condensación superficial o intersticial son valores no aportados por el software, conociendo las temperaturas de bulbo seco en que las temperaturas de rocío son sobrepasadas es posible determinar en gráficos de isotermas el punto en que ambas temperaturas entran en contacto y se inicia la condensación intersticial.

Con lo expuesto pueden reconocerse principalmente dos puntos críticos en el comportamiento higrotérmico y energético general de los sistemas constructivos no convencionales de uso habitual en la región. Por un lado, la inaptitud de los cerramientos respecto de los puentes térmicos, comprobada numérica y gráficamente con las dos metodologías propuestas. Esto se debe principalmente al descuido de la proporcionalidad entre valores de transmitancia térmica por la perse- cución de bajos niveles de conductividad térmica de los muros opacos $y$, por otro lado, riesgo constante de condensación intersticial, dado que los materiales aislantes térmicos, por lo general de naturaleza porosa, no impiden el paso de vapor de agua de igual manera que lo hacen con el paso del flujo de calor. A partir de este diagnóstico higrotérmico y energético, se propusieron medidas de optimización del diseño de los sistemas constructivos, en la búsqueda de mejorar sus comportamientos frente a la transmisión del calor y en especial atenuar el efecto de los puentes térmicos.

\section{Optimización}

Los criterios de optimización pueden inscribirse en dos líneas. Considerando que en cuatro de los cinco casos los puentes térmicos resultan estructuralmente imprescindibles, lo perseguido fue la reducción de la diferencia de transmitancia térmica entre muro opaco y puente térmico, para ajustarse a la tolerancia del $35 \%$ establecida por IRAM. Esto se consiguió por un lado reemplazando el material que constituia el puente térmico por uno de menorvalor deconductividad, sin modificar sus propiedades mecánicas. Por ejemplo, las armaduras de acero pudieron ser reemplazadas por varillas de resina de vinil y fibra de vidrio. Otra medida aplicada fue la sustitución del material aislante de baja conductividad térmica por otro de conductividad mayor, sin que el cerramiento en general pierda la clasificación obtenida respecto de IRAM 11.605 para las zonas bioambientales I y II. Así la espuma de poliuretano $(0,022 \mathrm{~W} /$ $\left.\mathrm{m}^{\circ} \mathrm{C}\right)$ o la lana de vidrio $(0,032 \mathrm{~W} /$ $\mathrm{m}^{\circ} \mathrm{C}$ ) pudieron ser reemplazadas por poliestireno expandido 0.035 $\left.\mathrm{W} / \mathrm{m}^{\circ} \mathrm{C}\right)$. Asimismo, se procedió a la reubicación de la porción aislante dentro de las capas del cerramiento. Se lo retiró de su ubicación usual en el espacio dejado por los entrama- 
dos estructurales y se lo ubicó por delante, lo que permitió cubrir y proteger con aislación térmica todo el cerramiento. Esto a su vez permitió dejar los intersticios estructurales rellenos de aire, de menor resistencia térmica que los materiales aislantes colocados anteriormente en la misma posición, y se redujo considerablemente la diferencia de conductividad térmica entre ambas porciones del cerramiento. Las diferentes capas constitutivas de la sección transversal deben asimismo mantenerse unidas entre sí, y para ello requieren elementos de fijación. Debido a esto, la continuidad estricta del aislante no siempre es posible, ya que se constituyen puentes térmicos no solo a efectos estructurales generales de la edificación, sino propios del cerramiento. Por ello se buscó reemplazar este tipo de situaciones por componentes que empleen sistemas de fijación sin necesidad de estructuras intermedias, como las placas de roca de yeso de terminación interior aplicadas como revoques secos con el uso de adhesivos, en vez de medios tabiques con estructura de perfiles galvanizados. Los sistemas constructivos totalmente industrializados, como los paneles prefabricados que en obra simplemente se ensamblan, son más propensos a adquirir este tipo de medidas por su producción más racionalizada y controlada.

Anteelsegundo punto crítico reconocido en los sistemas constructivos no convencionales, el siguiente criterio de optimización fue la disminución del riesgo de condensación intersticial mediante el freno del paso de vapor de agua a través del sistema constructivo, reubicando la barrera de vapor dentro de la multicapa o aumentando la resistencia a su paso. Esto último se logra mediante el incremento de la cantidad de barreras de vapor empleadas o el reemplazo de algunos materiales muy permeables al paso de vapor por otros de mayor resistencia. También se planteó la posibilidad de disminuir los saltos

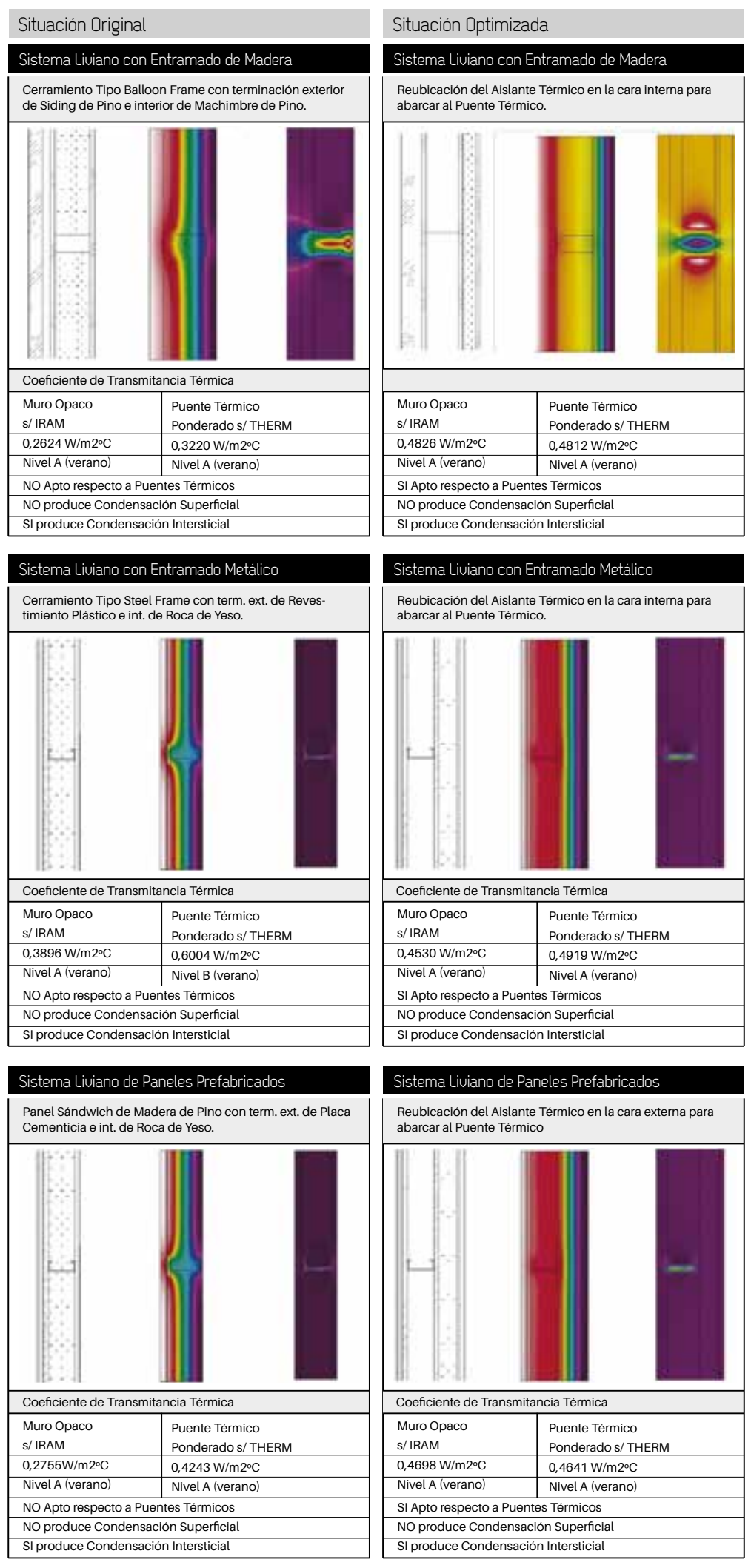

Tabla 1. Comparación de situación original y optimizada de los sistemas constructivos no convencionales analizados.

Fuente: elaboración propia 


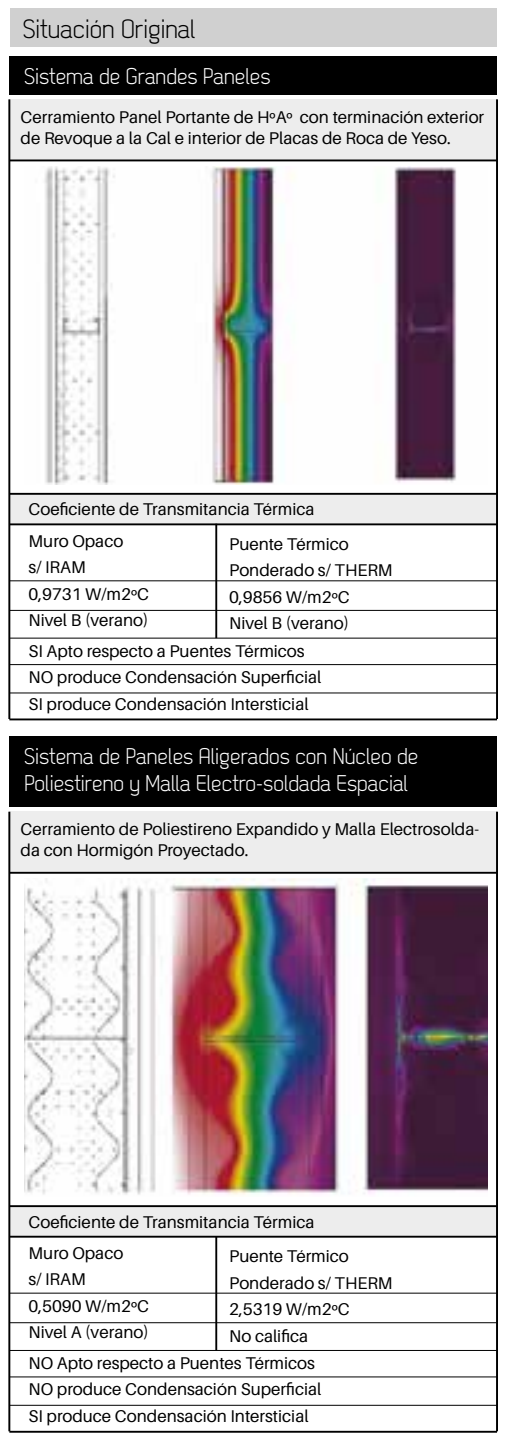

térmicos entre las diferentes capas constitutivas reduciendo el material o el espesor de la porción del aislante térmico, a fin de que las temperaturas de bulbo seco y de rocío no entren en contacto. La tabla 1 expone los resultados más relevantes de los sistemas estudiados con ambas metodologías y en los casos originales y optimizados. Los gráficosilustrados en cada situación corresponden a un detalle de la sección transversal del sistema en estudio (izquierda), a la escala colorimétrica de gradiente de temperatura (centro) y a la escala colorimétrica de magnitudes de flujo (derecha). En ambas escalas, los colores fríos (azules y morados) corresponden a temperaturas y flujos de calor bajos,
Situación Optimizada

\section{Sistema de Grandes Paneles}

Eliminación del Puente Térmico aplicando un Revoque Seco sobre Poliestireno Expandido.

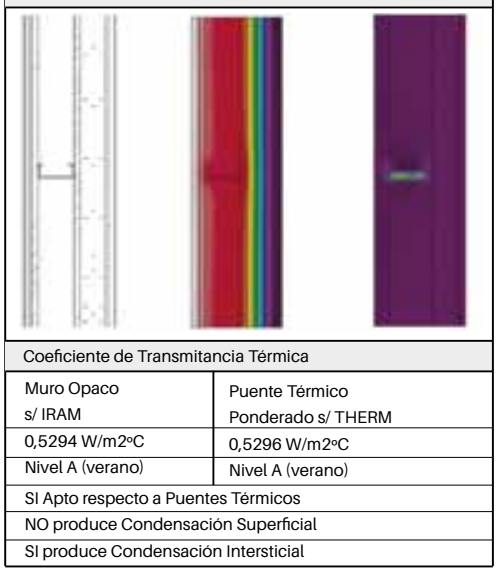

SI produce Condensación Intersticia

Sistema de Paneles Aligerados con Núcleo de Poliestireno y Malla Electro-soldada Espacial

Reemplazo del Acero por Varillas de Resina de Vinil y Fibra de Vidrio.

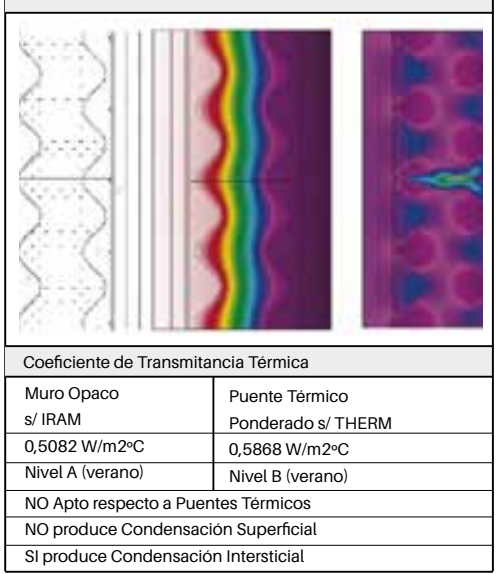

mientras los colores cálidos (amarillos y rojos) representan temperaturas y flujos de calor altos.

Como resultados más relevantes de las simulaciones de los sistemas constructivos en su situación optimizada respecto de la original, puede observarse que en todos los casos el gradiente de temperatura a través de la sección transversal se vuelve más regular. Esto responde a la atenuación del efecto del puente térmico y genera isotermas más paralelas que dejan de bordear la heterogeneidad. Asimismo, puede reconocerse que las mayores magnitudes de flujo de calor se redistribuyen, lo que da la pauta de que no solo el material utilizado
Tabla 1. Comparación de situación original y optimizada de los sistemas constructivos no convencionales analizados. Fuente: elaboración propia

es decisivo en el comportamiento térmico, sino que lo es también su ubicación respecto del conjunto.

Las variaciones en el valor de coeficiente ponderado de transmitancia térmica, por su lado, siguen distintas tendencias. En algunos casos, el valor en la situación optimizada disminuye respecto de la original, correspondiendo a la eliminación del puente térmico o al reemplazo del material altamente conductor térmico que lo conformaba por otro de menor poder de transmisión del calor. En otros casos, el valor aumenta en la situación optimizada. A simple vista esto podría percibirse como negativo, ya que las ganancias y pérdidas térmicas a través del cerramiento también se incrementarian. Este aumento, sin embargo, implica una disminución de la diferencia entre la transmitancia térmica de la porción de muro opaco y la del puente térmico, y en el 80 $\%$ de los casos se logra la aptitud respecto de los puentes térmicos, frente al $20 \%$ registrado en la situación original. Esto constituyó el objetivo principal propuesto por el presente trabajo, considerando que los puentes térmicos son elementos recurrentes en la construcción no convencional. No obstante, los valores de transmitancia térmica en la situación optimizada se mantienen dentro de los márgenes del nivel $\mathrm{A}$ (óptimo) propuesto por la Norma IRAM 11.605.

Otro fenómeno observado principalmente en los sistemas que emplean elementos de madera como estructura principal de los entrenados es que, al reubicar el material aislante, las heterogeneidades se convierten en los puntos del cerramiento en que se producen los menores flujos de calor, ya que, debido a su masa, ofrecen mayor 
resistencia al paso de temperatura que las cámaras de aire que se encuentran en sus intersticios en reemplazo del material aislante que lo ocupaba anteriormente. De esta manera, la lógica de muro opaco y puente térmico se invierte. En cuanto al segundo punto crítico, el riesgo de condensación intersticial no pudo ser erradicado por completo, pese a la aplicación de las estrategias de optimización. Se logró reducir la diferencia de temperatura de bulbo seco y de rocío y se retardó el proceso, pero la eliminación total del fenómeno únicamente se conseguiría con la implementación de soluciones costosas y constructivamente complejas, como la incorporación de numerosas barreras de vapor o la resignación de propiedadesaislantes térmicas del cerramiento.

\section{CONCLUSIONES Y REFLEXIONES FINALES}

El principio de racionalidad dota a los sistemas de construcción no convencional de empleo más usual en el NEA de consideraciones de confort ambiental y aislación térmica. Por ello registran buenos comportamientos higrotérmicos y energéticos generales que pudieron ser verificados con diferentes metodologías. Sin embargo, uno de los principales puntos críticos que pudo reconocerse en el presente trabajo fue la influencia negativa que tienen los puentes térmicos en el paso de energía calórica a través de las secciones transversales de estas tipologías constructivas, que por su naturaleza estructural cuentan con un gran número de ellos. Dejan de ser "anomalías" en el desarrollo de las envolventes para convertirse en elementos preponderantes. Por ello, en el momento de diseñar soluciones constructivas de este tipo es imprescindible buscar no solo la menorconductividad térmica posible en la porción opaca, sino estudiar la relación y proporcionalidad entre esta y los puentes térmicos.

También seconsidera muy importante evaluar las grandes disminuciones de temperatura que producen los materiales aislantes térmicos dentro del cerramiento en relación con el principal flagelo que acusa a la construcción en la región, la condensación intersticial de vapor de agua. Esta problemática, sin embargo, se cree sobreestimada por la normativa IRAM por los valores de diseño excesivamente bajos que determina para las verificaciones en la región.

Las consideraciones aquí mencionadas y las propuestas de optimización de los sistemas constructivos planteadas y verificadas pretenden ser aplicadas por los diferentes diseñadores en la región. Sin embargo, se deben acompañar por una mayor exigencia y control en el cumplimiento efectivo de la normativa de habitabilidad y técnico-constructiva vigentes por parte de los diferentes estamentos gubernamentales, ya que su aplicación se limita en la mayoría de los casos únicamente a voluntades expresas de diseñadores, constructores o comitentes. En especial, si se considera que las mejoras en los rendimientos de los diferentes sistemas constructivos significan en la mayoría de los casos aumentos de los costos iniciales, los únicos que por lo general son tomados en cuenta, dejando de lado las disminuciones en costos de mantenimiento a largo plazo que se lograrían. En una industria tan competitiva como la construcción, ello genera pérdida de competitividad si no todos los actores aplican o son obligados a aplicar iguales criterios de diseño.

Por otro lado, los reglamentos y códigos de construcción de las principales ciudades de la región deben ser revisados, ya que se encuentran muy retrasados respecto de los importantes avances que evidencia la construcción industrializada, con el objetivo de una inclusión más amplia de consideraciones respecto de las performances higrotérmicas y energéticas de los sistemas constructivos en general y de la normalización del uso de los sistemas constructivos no convencionales. Todo en favor de un cambio de paradigma que tome en cuenta principalmente costos económicos y ambientales de todo el ciclo de vida de las edificaciones, no solo las inversiones iniciales, en especial ante la crisis ecológica mundial y el déficit energético que vive la región.

El desarrollo de la investigación, asimismo, permitió la familiarización con un nuevo mecanismo disponible para las tareas de evaluación higrotérmica de las envolventes perimetrales de las edificaciones en general. Especificamente el software de simulación THERM 6.3 se encontró como una herramienta muy útil y confiable para el cálculo de la transmitancia térmica de las secciones transversales. Posee una interface sencilla y arroja valores muy similares a aquellos obtenidos mediante las metodologías propuestas por la normativa de habitabilidad vigente en la Argentina. Sin embargo, no suplanta a estas, ya que, dada la 
naturaleza de los cálculos y resultados, para poder establecer iguales relaciones que con la Norma IRAM ( $\mathrm{Kpt} / \mathrm{Kmo}$ ) debe desarrollarse una serie adicional de operaciones que terminan siendo más engorrosas y largas que la aplicación de las planillas de cálculo que propone el organismo argentino. El software tampoco calcula los Riesgos de Condensación Superficial e Intersticial de los diferentes cerramientos, verificaciones muy importantes en la determinación de sus comportamientos higrotérmicos. Estas diferencias esenciales impiden que los resultados de las simulaciones puedan relacionarse directamente con estándares, clasificaciones y especificaciones determinadas por IRAM. Otra desventaja que presenta la aplicación de THERM, considerando usuarios de habla no inglesa, es que en la actualidad el software y sus manuales de uso únicamente se encuentran disponibles en inglés. Esta situación se agudiza considerando que el actual plan de estudios de la Carrera de Arquitectura de la Facultad de Arquitectura y Urbanismo de la Universidad Nacional del Nordeste no incluye el idioma como materia curricular, lo que limita la posibilidad de aprovechamiento de estudiantes y profesionales del medio de una herramienta de tan fácil acceso como este software.

A pesar de las diferencias descriptas anteriormente, se reconocen dos grandes ventajas en la aplicación de THERM. En primer lugar, la posibilidad de obtener un Coeficiente Ponderado de Transmitancia Térmi$c a$, que incorpora ambas porciones del cerramiento y determina un valor de transmitancia térmica promedio que puede aplicarse a toda la envolvente, sin caer en errores de consideración exclusiva del muro opaco para la clasificación de los sistemas. En segundo lugar, se considera una herramienta muy útil para diseñadores por proveer información muy valiosa de orden gráfico. Esta información no es aportada por IRAM y es de gran utilidad para el reconocimiento claro del comportamiento de las secciones transversales de los cerramientos y en especial de la influencia que ejercen los puentes térmicos en el comportamiento higrotérmico y energético.
Se concluye entonces que la importante presencia de puentes térmicos en la construcción no convencional ejerce una gran influencia en el comportamiento higrotérmico y energético de estas soluciones constructivas, y que ellos pueden transformarse en verdaderos problemas crónicos, si se alejan los comportamientos reales de aquellos estudiados teóricamente, en especial si se realiza una aplicación parcial de las normativas de habitabilidad y demás metodologías de evaluación. Los puentes térmicos deben ser considerados elementos principales y no excepciones en la construcción no convencional y hay que tratar de mitigarlos desde el proceso de diseño de sistemas industrializados que contemplan principios de racionalidad, mecanización y automatización.

Finalmente, la visualización gráfica clara de sus comportamientos mediante software de simulación como el empleado en el presente trabajo contribuye en gran medida a comprender e interpretar sus influencias.

\section{CITAS Y REFERENCIAS BIBLIOGRÁFICAS}

GARCÍA MARQUINA, E. (2013) Estudio-Diagnóstico sobre las Posibilidades del Desarrollo de una Edificación Residencial Industrializada dirigida a satisfacer las Necesidades de Vivienda Pública y muy especialmente en Alquiler en la Comunidad Autónoma del País Vasco. Programa "Eraikal". España.

NORMA IRAM 11.549. Aislamiento térmico de edificios. Vocabulario. Tercera Edición. Instituto Argentino de Racionalización de Materiales. Buenos Aires, Argentina. LAWRENCE BERKLELEY NATIONAL LABORATORY
(2013). THERM 6.3 / WINDOW 6.3 NFRC Simulation Manual. Berkeley, California, Estados Unidos. Disponible en https://windows.lbl.gov/software/NFRC/SimMan/ NFRCSim6.3-2013-07-Manual.pdf.

MAC DONNEL, H. (1999) Manual de Construcción Industrializada. Editorial Revista Vivienda SRL. Buenos Aires, Argentina.

SARMANHO FREITAS, A. M. y MORAES DE CASTRO, R. C. (2007) Steel Framing: Arquitectura. Instituto Latinoamericano del Fierro y el Acero. Santiago de Chile, Chile. 